\title{
Incidência de LER/DORT em funcionários do setor de Engenharia da Fundação Nacional de Saúde
}

\author{
Incidence of RSI/WRMD sector employees in Engineering \\ from the National Health Foundation \\ Incidencia de LER / DORT en funcionarios del sector de Ingeniería \\ de la Fundación Nacional de Salud \\ Janaina Cardoso dos SANTOS ${ }^{1}$ \\ Hellen Cristina Barbosa PEREIRA ${ }^{2}$ \\ ${ }^{1}$ Fisioterapeuta pela Associação de ensino Renovado, ASPER, 58031-130 João Pessoa - PB, Brasil. \\ ${ }^{2}$ Fisioterapeuta pela Faculdade Maurício de Nassau, UNINASSAU, 58030-000, João Pessoa - PB, Brasil
}

\begin{abstract}
Resumo
As LER/DORT são doenças de origem ocupacional causadas por movimentos repetitivos. Uma das mais importantes causas de afastamento do trabalho e tem como principal sintomatologia a dor. Ciente da gravidade desse problema foi realizada esta pesquisa com o objetivo de verificar a incidência de LER/DORT em funcionários no setor de engenharia da Fundação Nacional de Saúde (FUNASA), com a finalidade de estes dados servirem de alerta para os cuidados da qualidade de vida laboral dos trabalhadores da amostra. Trata-se de um estudo transversal de abordagem descritiva, exploratória e quantitativa. A amostra se constituiu de 32 funcionários do setor de Engenharia da Fundação Nacional de Saúde na cidade de João Pessoa. Utilizou-se um questionário com perguntas sobre o perfil dos profissionais, sintomas do estresse e avaliação do ambiente de trabalho. Foi observado que as condições ambientais no trabalho Ruído, temperatura, iluminação não afetam o rendimento no trabalho. A idade dos funcionários varia entre 17 a 61 anos, sendo 37,5\% ( $\mathrm{n}=12$ ) do sexo feminino e $62,5 \%(n=20)$ do sexo masculino. Destes $46,8 \%(n=15)$ praticam algum tipo de atividade física, e $53,1 \%(\mathrm{n}=17)$ não praticam. Foi observado que a maioria dos profissionais trabalha na posição sentada 71,9\%, $(\mathrm{n}=23)$ e dentre a sintomatologia dolorosa apresentada, apontou a coluna lombar com a maior incidência, sendo 52,6\% ( $\mathrm{n}=10)$. Os resultados obtidos ainda mostram o quanto são necessárias mudanças ergonômicas no ambiente de trabalho, na busca de promover uma melhor qualidade de vida dos profissionais, minimizando a ocorrência de dor.

Descritores: Transtornos Traumáticos Cumulativos; Prevenção \& Controle; Engenharia Humana.
\end{abstract}

\begin{abstract}
RSI / WRMD are occupational diseases caused by repetitive movements. One of the most important causes of withdrawal from work and its main symptomatology is pain. Aware of the severity of this problem, this research was carried out in order to verify the incidence of RSI / WRMD in employees in the engineering sector of the National Health Foundation (FUNASA), in order to serve as an alert for quality Working life of the sample workers. This is a cross-sectional, descriptive, exploratory and quantitative study. The sample consisted of 32 employees from the engineering sector of the National Health Foundation in the city of João Pessoa. A questionnaire was used with questions about the profile of the professionals, symptoms of stress and evaluation of the work environment. It has been observed that environmental conditions at work Noise, temperature, lighting do not affect the performance at work. The age of employees varies from 17 to 61 years, with $37.5 \%(n=12)$ female and $62.5 \%(n=20)$ males. Of these, $46.8 \%(n=15)$ practiced some type of physical activity, and $53.1 \%(\mathrm{n}=17)$ did not practice. It was observed that most of the professionals work in the seated position $(\mathrm{n}=23)$, and among the pain symptomatology presented, the lumbar spine showed the highest incidence, being $52.6 \%(n=10)$. The results still show how much ergonomic changes are needed in the work environment, in the search to promote a better quality of life of the professionals, minimizing the occurrence of pain.
\end{abstract}

Descriptors: Cumulative Trauma Disorders; Prevention \& Control; Human Engineering.

\section{Resumen}

Las LER / DORT son enfermedades de origen ocupacional causadas por movimientos repetitivos. Una de las causas más importantes de alejamiento del trabajo y tiene como principal sintomatología el dolor. La investigación con el objetivo de verificar la incidencia de LER / DORT en funcionarios en el sector de ingeniería de la Fundación Nacional de Salud (FUNASA), con el fin de que estos datos sirven de alerta para el cuidado de la calidad de La vida laboral de los trabajadores de la muestra. Se trata de un estudio transversal de enfoque descriptivo, exploratorio y cuantitativo. La muestra se constituyó de 32 funcionarios del sector de Ingeniería de la Fundación Nacional de Salud en la ciudad de João Pessoa. Se utilizó un cuestionario con preguntas sobre el perfil de los profesionales, síntomas del estrés y evaluación del ambiente de trabajo. Se observó que las condiciones ambientales en el trabajo Ruido, temperatura, iluminación no afectan el rendimiento en el trabajo. La edad de los empleados varía entre 17 a 61 años, siendo el 37,5\% $(\mathrm{n}=12)$ del sexo femenino y el 62,5\% $(\mathrm{n}=20)$ del sexo masculino. De estos 46,8\% $(\mathrm{n}=15)$ practican algún tipo de actividad física, y $53,1 \%(\mathrm{n}=17)$ no practican. Se observó que la mayoría de los profesionales trabajan en la posición sentada 71,9\%, $(\mathrm{n}=$ 23) y entre la sintomatología dolorosa presentada, apuntó la columna lumbar con la mayor incidencia, siendo el 52,6\% (n $=10$ ). Los resultados obtenidos todavía muestran cuán necesarios son necesarios cambios ergonómicos en el ambiente de trabajo, en la búsqueda de promover una mejor calidad de vida de los profesionales, minimizando la ocurrencia de dolor. Descriptores: Trastornos de Traumas Acumulados ; prevención \& control; Ingeniería Humana.

\section{INTRODUÇÃO}

Os Distúrbios Osteomusculares Relacionados ao Trabalho (DORT) ou Lesões por Esforços Repetitivos (LER) são termos de origem ocupacional decorrentes de distúrbios funcionais que implicam em fadiga localizada nos nervos, músculos, tendões, sinóvias, fáscias e ligamentos ${ }^{1}$. A LER/DORT é uma das mais importantes causas de afastamento e destaca-se entre as maiores repercussões na saúde do trabalhador decorrentes das transformações do trabalho, principalmente dos novos modelos organizacionais e de gestão ${ }^{2}$. 
A LER/DORT está relacionada à biomecânica corporal, onde a posição incorreta das estruturas osteomusculares (músculo, tendões, fáscias e nervos) geram consequências como: dor, fadiga, queda do rendimento no trabalho e incapacidade temporária, podendo causar uma síndrome dolorosa crônica ${ }^{3}$.

Alguns fatores relacionados à causalidade estão diretamente ligados ao aparecimento dos DORTs no ambiente de trabalho que são eles: Repetitividade; Postura inadequada; Força Excessiva; Compressão; Vibração Mecânica e Predisposição ${ }^{4}$.

As DORT progridem de forma homogênea relativamente aos estágios evolutivos, sendo reconhecidos em quatro estágios ${ }^{5}$ :

Grau I: (primeiro estágio) tem como principal característica uma sensação de peso e desconforto no membro ou região afetada, dor em pontada que piora com o trabalho e melhora com o repouso, permanece com a capacidade produtiva normal, ausência de sinais clínicos. Bom prognóstico com tratamento adequado.

Grau II: se dá a maior incidência de procura por atendimento e é caracterizado por dor tolerável, porém, mais persistente e intensa. A dor é mais localizada com sensações de calor e formigamento, podendo acontecer alterações da sensibilidade tátil. Os sinais clínicos ainda encontram-se ausentes ou poucos pronunciados a palpação da musculatura pode revelar aumento da resistência tecidual e dor. O prognóstico é favorável.

Grau III: é caracterizada por dor persistente e forte, pouco suavizada com o repouso (apenas diminui a intensidade da dor), com irradiação mais definida, diminuição de força muscular, perda do controle dos movimentos, alterações de sensibilidade (quase sempre presentes), palidez, hiperemia, sudorese, a mobilização ou a palpação da região acometida provoca dor forte redução da produtividade ou impossibilidade de executar funções. Prognóstico reservado.

Grau IV: (último estágio) ocorre dor forte, contínua e as vezes insuportável, levando o indivíduo a um sofrimento intenso. Mesmo os movimentos mais suaves aumentam o quadro doloroso que se irradia por todo o segmento, há diminuição da força e constantemente perda dos movimentos, edema persistente, são comuns deformidades e atrofias, incapacidade de desenvolver tarefas do trabalho ou domésticas, há quadros de depressão, ansiedade e angústia. O prognóstico é sombrio.

A maior incidência está entre os trabalhadores jovens e as mulheres, sendo essa ultima justificada por questões hormonais, pela dupla jornada de trabalho, pela falta de preparo muscular para algumas tarefas e também pelo número de mulheres no mercado de trabalho ter crescido consideravelmente ${ }^{6}$.

Diante de tal problemática, durante a Segunda Guerra Mundial foi desenvolvida a ergonomia, quando existiu a necessidade de adequações do homem às maquinarias da época, já que os equipamentos utilizados exigiam de seus operadores repostos rápidas e realização de novas atividades em situações críticas ${ }^{5}$.

A ergonomia é uma ciência que visa melhorar a segurança, saúde, conforto e eficiência no trabalho, enfocando o homem, avaliando as condições de insegurança, desconforto, ineficiência, eliminando-os e adaptando as capacidades e limitações físicas e psicológicas do homem, estudando vários aspectos como a postura e os movimentos do corpo, fatores ambientais como ruídos e agentes químicos, tendo a intenção de tornar os ambientes mais seguros, saudáveis, confortáveis e eficientes, tanto no trabalho como na vida cotidiana, pois todos esses fatores podem interferir na saúde, a segurança e no conforto deste trabalhador ${ }^{7}$.

Os benefícios diretos da ergonomia incluem a redução dos riscos de acidentes e de doenças ocupacionais, redução da frequência, contratações e custos de treinamento, eliminação de custos de redesenho de postos de trabalho e de equipamentos dos incidentes, redução de indenizações e custos com tratamentos de saúde. Já os indiretos são a melhoria da qualidade de vida do trabalho para os funcionários, redução da perda de tempo e das incapacitações, melhoria da saúde do trabalhador e do conforto, aumento da qualidade do trabalho e da produtividade, melhoria da satisfação e da moral do trabalhador, motivação do trabalhador, redução do estresse no ambiente de trabalho, trabalho adaptado para pessoas portadoras de deficiência, projeto de modificação de trabalhos pesados ${ }^{8}$.

Além da melhoria no ambiente de trabalho, foi implantado a ginastica laboral que é uma das medidas tomadas para a prevenção e reabilitação de LER/DORT, além de ser uma iniciativa que tem por objetivo promover a saúde e a qualidade de vida do trabalhador'.

Diante de tal problemática, a pesquisa se justifica pela necessidade de se fazer uso dos princípios ergonômicos para facilitar o trabalho e modificar o ambiente e ritmo de trabalho, além de se avaliar a presença de estresse e Distúrbios Osteomusculares Relacionados ao Trabalho, procurando enfocar a aplicação desses princípios para beneficio dos funcionários, melhorando assim as condições de saúde e repercutindo na produtividade da empresa.

Pelo exposto, este estudo visa detectar a incidência de LER/DORT em funcionários do setor de engenharia da Fundação Nacional de Saúde, com a finalidade de estes dados contribuírem para a qualidade de vida laboral dos trabalhadores desta repartição.

\section{MATERIAL E MÉTODO}

Trata-se de um estudo transversal de abordagem descritiva, exploratória e quantitativa. Descritiva, pois é um tipo de estudo mais apropriado para o pesquisador que busca ter um melhor entendimento sobre determinado fenômeno, possibilitando ao pesquisador um bom desenvolvimento mais sem interferir na realidade do fenômeno. Também é um estudo exploratório que tem como objetivo a elaboração de hipóteses e proporcionar maior intimidade com o problema, visando torná-lo mais claro. E por último, é quantitativo, pois fez uso da quantificação de dados e de recursos estatísticos (números) para analisá-los buscando sempre uma variável ${ }^{10}$.

A população foi composta por engenheiros, técnico em cartografia, auxiliar de saneamento, agente administrativo, agente de saúde pública, ecônomo, auxiliar de administração e estagiários do setor de Engenharia da Fundação Nacional de saúde (FUNASA) e a amostra por acessibilidade e conveniência, foi composta por 32 dos 39 funcionários locados nesta repartição.

A pesquisa foi realizada na Fundação Nacional de Saúde (FUNASA) do município de João Pessoa- PB, no período de Junho de 2011. A autorização para realização do trabalho foi concedida mediante assinatura de documento pelo superintendente da instituição.

Foi utilizado um questionário elaborado pela pesquisadora, no qual foram abordadas questões sobre o perfil dos profissionais, incluindo idade e sexo, além de 
perguntas sobre a sintomatologia dolorosa, posto de trabalho, estresse, condições ambientais e o índice de sintomas osteomusculares nos trabalhadores da amostra.

Foi aplicado um termo de consentimento livre e, onde os avaliados foram informados do sigilo das informações prestadas, atendendo a resolução 196/96. Os questionários foram aplicados após serem aprovados pelo comitê de ética. De posse dos dados esses foram analisados mediante programa Excel.

\section{RESULTADOS E DISCUSSÃO}

De acordo com a verificação do posto de trabalho no setor de engenharia da Fundação Nacional de Saúde (FUNASA), verificou-se que os funcionários executam suas atividades laborais em postos de trabalho fixo, na posição sentada, sendo 71,9\% (n=23) da amostra. A postura sentada na maioria das vezes, não resulta em um relaxamento da musculatura do corpo e num trabalho mais fácil e confortável. Só conseguimos condições adequadas nesta postura quando o mobiliário é corretamente adequado às características do usuário ${ }^{11}$.

Diante do exposto, foi observado que a altura da mesa utilizada pelos funcionários está em condições ergonômicas inadequadas forçando o usuário a fazer uma cifose torácica, é observado também à falta de apoio adequado para os pés e braços, porém, os funcionários deslocam-se sempre que necessário.

Quanto ao uso do computador, é observado que o equipamento não esta em um bom ângulo de visão, deve-se posicionar o monitor à altura dos olhos para prevenir dores no pescoço e ombro. Ter um adequado uso do computador ajudará o usuário a se sentir mais consciente de que seu organismo não é uma máquina e que por isso, precisa de cuidados constantes. Em geral, são encontrados: mesas para o uso do computador e avaliação de documentos (Figura 1).

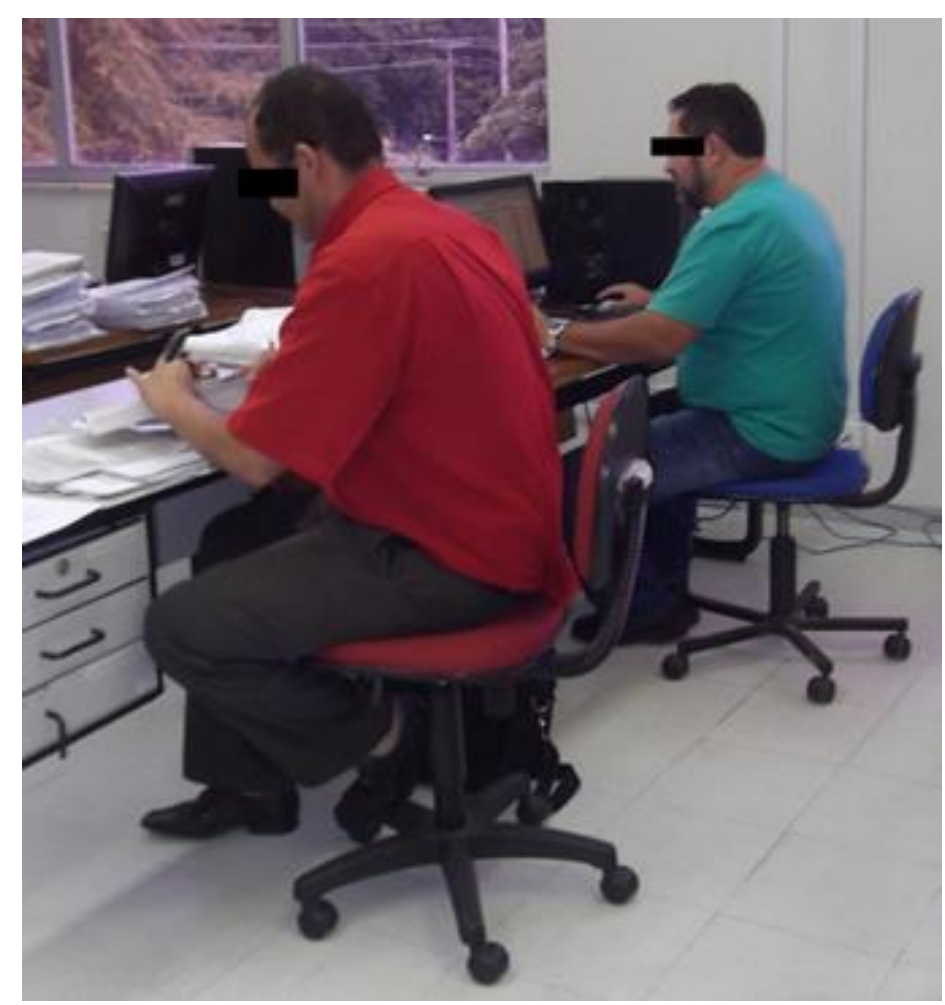

Figura 1: Funcionário em seu posto de trabalho. Fonte: Dados da pesquisa, 2011.

As idades variaram entre 17 e 61 anos, sendo 12 do sexo feminino representado $37,5 \%$ da amostra e 20 do sexo masculino totalizando $62,5 \%$ da amostra em um número total de 32 questionários aplicados.

Destes, foi verificado que 15 praticam algum tipo de atividade física, sendo $46,8 \%$ da amostra, e que 17 pessoas não praticam atividades físicas totalizando $53,1 \%$ da amostra. A prática de atividade física de forma regular e controlada apresenta-se, como um importante meio de mantermos em atividade nossos sistemas orgânicos, permitindo seu melhor funcionamento, diminuindo o risco de algumas doenças e proporcionando-nos uma maior aptidão da qualidade de gordura corporal e do seu padrão de distribuição. Está comprovado que quanto mais ativo o indivíduo, menos limitações físicas ele terá ${ }^{12}$.

Foram feitas perguntas relacionadas ao estresse para saber o nível que cada item pode afetar ou não as pessoas no ambiente de trabalho, indicando algum risco futuro para a saúde dos funcionários (Gráfico 1). Os itens abordados não foram suficientes para afetar a satisfação com o trabalho, dos 32 questionários aplicados 93,75\% ( $\mathrm{n}=30)$ relataram sentir-se satisfeito em seu trabalho.

O estresse causa ao individuo exigências inatingíveis, trazendo baixo desempenho e níveis moderados de estresse podem com o tempo se tornar um agravante no desempenho do indivíduo ${ }^{13}$.

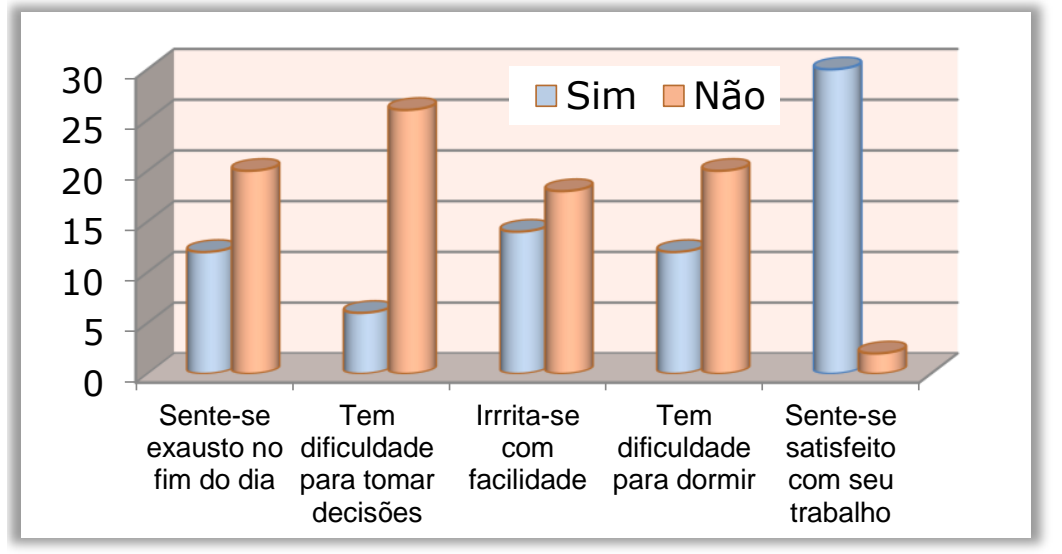

Gráfico 1: Distribuição quanto aos sintomas de estresse.

No que diz respeito às condições ambientais no trabalho foi possível observar que, 34,4\% $(\mathrm{n}=11)$ afirmaram ser baixo, 28,1\% (n=9) suportável, 25\% $(n=8)$ audível e $12,5 \% \quad(\mathrm{n}=4)$ alto. Diante da afirmação supracitada observou-se que a caracterização dos ruídos no ambiente em que trabalham em geral foi de baixa intensidade, não prejudicando a saúde dos trabalhadores.

O ruído acima do esperado é incluído como um dos fatores mais agravantes de agressão nos locais de trabalho. Os ruídos intensos tendem a prejudicar tarefas que exigem concentração mental e certas tarefas que exigem atenção ${ }^{4}$. A respeito das condições ambientais do trabalho, é necessário aumentar a intensidade luminosa à medida que o contraste diminui e se exige a percepção de pequenos detalhes. Os locais de trabalho devem fornecer condições climáticas favoráveis ao desempenho do trabalhador ${ }^{7}$. Foi observado que $50 \%(\mathrm{n}=16)$ dos 32 participantes afirmaram que a temperatura do ambiente de trabalho é boa, 37,5\% (n=12) relataram ser excelente, $9,4 \%(n=3)$ suficiente e $3,1 \%(n=1)$ regular. Diante das informações colhidas conclui-se que as fontes de luz do local onde trabalham são de boa iluminação.

Em relação ao desconforto dos móveis e o da postura onde foi obtido que, $43,75 \%(n=14)$ dos funcionários que responderam os questionários acharam os móveis desconfortáveis e 56,25\% ( $\mathrm{n}=18)$ não acharam. Quanto à postura $46,8 \% \quad(n=15)$ apresentaram algum desconforto postural em oposição a $53,1 \%(n=17)$ que relataram não apresentar nenhum tipo desconforto.

Trabalhadores que estão sujeitos a condições ambientais impróprias no local de trabalho, como desconforto de postura e móveis desconfortáveis, são mais atingidos por dores habituais, dormência, formigamento ou inchaço em alguma parte do corpo ${ }^{14}$. 
Sobre as principais lesões decorrentes do trabalho e sintomatologia dolorosa 90,6\% $(n=29)$ relatam nunca ter tido afastamento em decorrência de lesão relacionada ao trabalho e 9,3\% (n=3) alegaram já ter se afastado por apresentar algum tipo de lesão. Entre as pessoas que relataram ter se afastado do trabalho $66,6 \%(n=2)$ tiveram tendinite e $33,3 \% \quad(n=1)$ lombalgia. A presença de movimentos repetidos, por si só, não é suficiente para produzir lesões ou para desencadear os fenômenos clínicos associados às LER/DORT ${ }^{14}$.

O Gráfico 2 mostra que entre os 32 entrevistados, $59,3 \% \quad(\mathrm{n}=19)$ relataram sentir desconforto muscular enquanto 40,6\% ( $n=13)$ não sentem nenhum tipo de dor.

No Gráfico 3 verificou-se a maior incidência de dor na coluna lombar. Dos 19 funcionários que apresentaram algum tipo de dor, $52,6 \%(\mathrm{n}=10)$ relataram ser na coluna lombar. Já no Gráfico 4 foi avaliado o nível de conhecimento a respeito da Cinesioterapia Laboral onde dos 32 avaliados $87,5 \%(n=28)$ não conheciam seus benéficos para saúde. O que é ressaltado através da publicação feita pela Fundação Emílio Odebrecht de 1985, onde relata que no Brasil, apenas $1 \%$ do prêmio seguro que os operários pagam ao INSS é utilizado em medidas preventivas, enquanto $99 \%$ se destinam a medidas curativas ${ }^{14}$. Assim, conclui que no Brasil usa-se o modelo de atendimento médico que objetiva a prevenção secundária e não a prevenção primária e a promoção de saúde ${ }^{15}$.

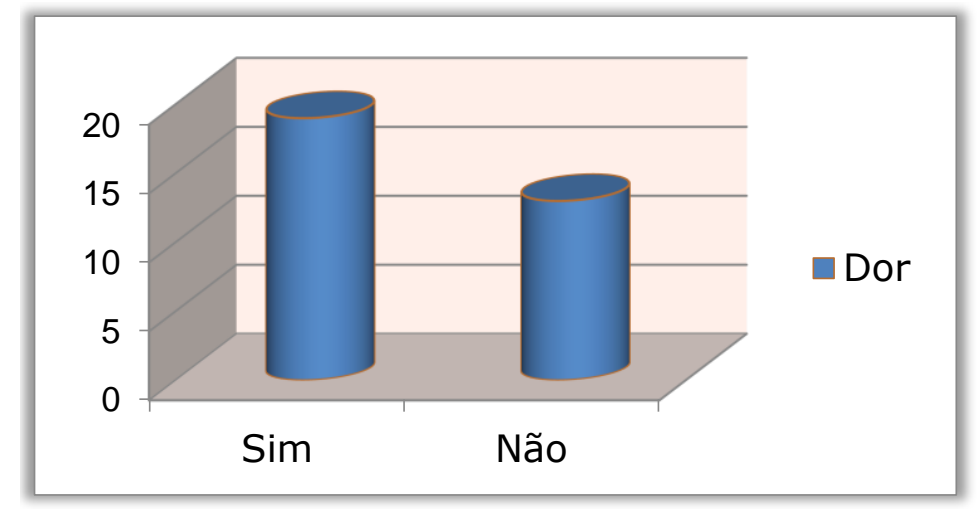

Gráfico 2: Distribuição quanto ao sintoma da dor.

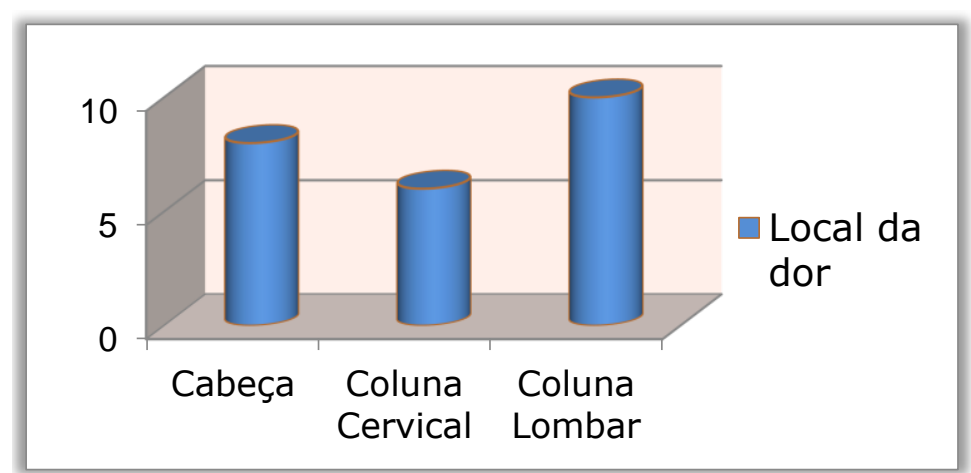

Gráfico 3: Distribuição dos principais locais que apresentam dor ou desconforto muscular.

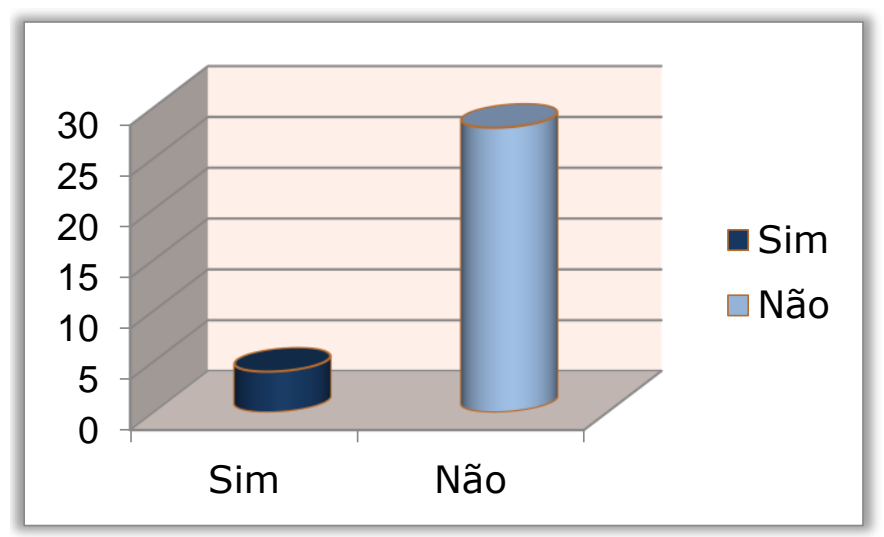

Gráfico 4: Distribuição quanto o conhecimento dos Benefícios da cinesioterapia laboral.

\section{CONCLUSÃO}

A partir da pesquisa realizada, observou-se que os funcionários executam suas atividades laborais em postos de trabalho fixo, na posição sentada, foi observado também que a altura da mesa utilizada pelos funcionários da FUNASA está em condições ergonômicas inadequadas. A idade dos funcionários varia entre 17 a 61 anos, sendo $37,5 \%(n=12)$ do sexo feminino e $62,5 \%(n=20)$ do sexo masculino. Destes $46,8 \%(n=15)$ praticam algum tipo de atividade física, e $53,1 \%(\mathrm{n}=17)$ pessoas não praticam atividades físicas.

Com base nos resultados obtidos constatou-se que a maioria dos profissionais observados apresenta sintomas de dor e desconforto muscular, a sintomatologia verificada apontou a coluna lombar com a maior incidência de queixa de dor e a prevalência do sedentarismo em funcionários da amostra.

Em relação às condições ambientais no trabalho ruído, temperatura, iluminação não afetam o rendimento no trabalho. Observou-se também o alto índice de satisfação com o trabalho, do baixo nível de estresse, além da falta de conhecimento a respeito da cinesioterapia laboral.

Através da verificação do posto de trabalho, observou-se que o mobiliário disponibilizado pela empresa não são adequados e não contribuem para o conforto dos funcionários.

O estudo trouxe algumas limitações, como a falta de alguns funcionários que complementaria os $100 \%$ locados no setor de engenharia da repartição, então, a amostra foi por acessibilidade e conveniência.

Conclui-se que, através da subjetividade do resultado do questionário aplicado, fazem-se necessárias mudanças ergonômicas de trabalho, na busca de promover uma melhor qualidade de vida dos profissionais, minimizando a ocorrência de dor.

Com base nos dados apresentados, sugerem-se as seguintes medidas: que novos estudos sejam realizados, a adoção de um programa de prevenção, com ginástica laboral, pausa, exercícios respiratórios, e alongamentos musculares, a contratação de um profissional da área da saúde, um fisioterapeuta, que participe na elaboração do programa de exercícios, na adequação do mobiliário e aquisição de materiais que busque a adaptação ergonômica ao posto de trabalho.

\section{REFERÊNCIAS}

1. Viudes PLR. LER e DORT no trabalho. Revista eficaz, Maringá, $2010 . \quad$ Disponível em:<http://www.institutoeficaz.com.br/revistacientifica/ wpcontent/uploads/2009/12/Patr\%C3\%ADcia-LessaViudes.pdf>, acesso em: 15. Março. 2011.

2. Mendes LF, Lancman S. Reabilitação de pacientes com LER/DORT: contribuições da fisioterapia em grupo. Rev bras saúde ocup. 2010; 35 (121):23-32.

3. Oliveira JRG. A Importância da Ginástica Laboral na Prevenção de Doenças Ocupacionais. 2007. Disponível em<http://www.cdof.com.br/artigo\%20g.\%20labora.pdf $>$. Acesso em 20. Março. 2011.

4. Barbosa LG. Fisioterapia preventiva nos distúrbios osteomoleculares relacionados ao trabalho - DORTs: a fisioterapia do trabalho aplicada. $2^{\mathrm{a}}$ ed. Rio de Janeiro: Guanabara Koogan; 2009.

5. Deliberato PCP. Fisioterapia Preventiva: Fundamentos e Aplicações. São Paulo: Manole, 2002. 
6. Spinelli JLM, Sales MdeP. Efeitos da cinesioterapia laboral Compensatória nas lombalgias de operadoras de checkout, Belém, 2006. Disponível em: $<$ http://www.unama.br/graduacao/cursos/Fisioterapia/tc c/2006/efeitos-da-cinesioterapia-laboral-compensatorianas-lombalgias-de-operadoras-de-checkout.pdf $>$, acesso em: 14. março.2011.

7. Dul J, Weerdmeester B. Ergonomia Prática. 2. ed. Edgar Blucher: São Paulo; 2004.

8. Iida I. Ergonomia: projeto e produção. 7. ed. São Paulo: Blücher; 2001.

9. Mendes RA, Leite N. Ginástica Laboral: Princípios e Aplicações Práticas. 2. ed. São Paulo: Manole; 2004.

10. Oliveira SL de. Tratado de Metodologia Científica. 2 ed. São Paulo: Thomson Pioneira; 1999.

11. Nascimento NM do, Moraes RA. Fisioterapia nas empresas: saúde x trabalho. 3. ed. Rio de Janeiro: Taba Cultural; 2000.

12. Fleck SJ, Kraemer WJ. Fundamentos do treinamento de força muscular. 2. ed. Porto Alegre: Artmed; 1999.

13. Robbins SP. Comportamento Organizacional. Rio de Janeiro: JC; 1999.

14. Oliva ACI. Abordagem fisioterapêutica na síndrome do túnel do carpo decorrente da ler, dort em mulheres. 2007. Disponivel em: <http://www.uva.br/ cursos/graduacao/ccbs/fisioterapia_monografias/aborda gem_fisioterapeutica_sindrome_tunel_carpo.pdf >, Acesso em:04, Junho, 2011.

15. Brasil, Ministerio da Educação; Secretaria do Educação Física e desporto. Esporte e Lazer na Empresa. Brasília: MEC / SEED, 1990.

\section{CONFLITO DE INTERESSES}

Os autores declaram não haver conflitos de interesse.

\section{AUTOR PARA CORRESPONDÊNCIA}

Janaína Cardoso dos Santos

ina-cardosos@hotmail.com

Submetido em 25/08/2017

Aceito em 28/09/2017 